Provided for non-commercial research and education use. Not for reproduction, distribution or commercial use.

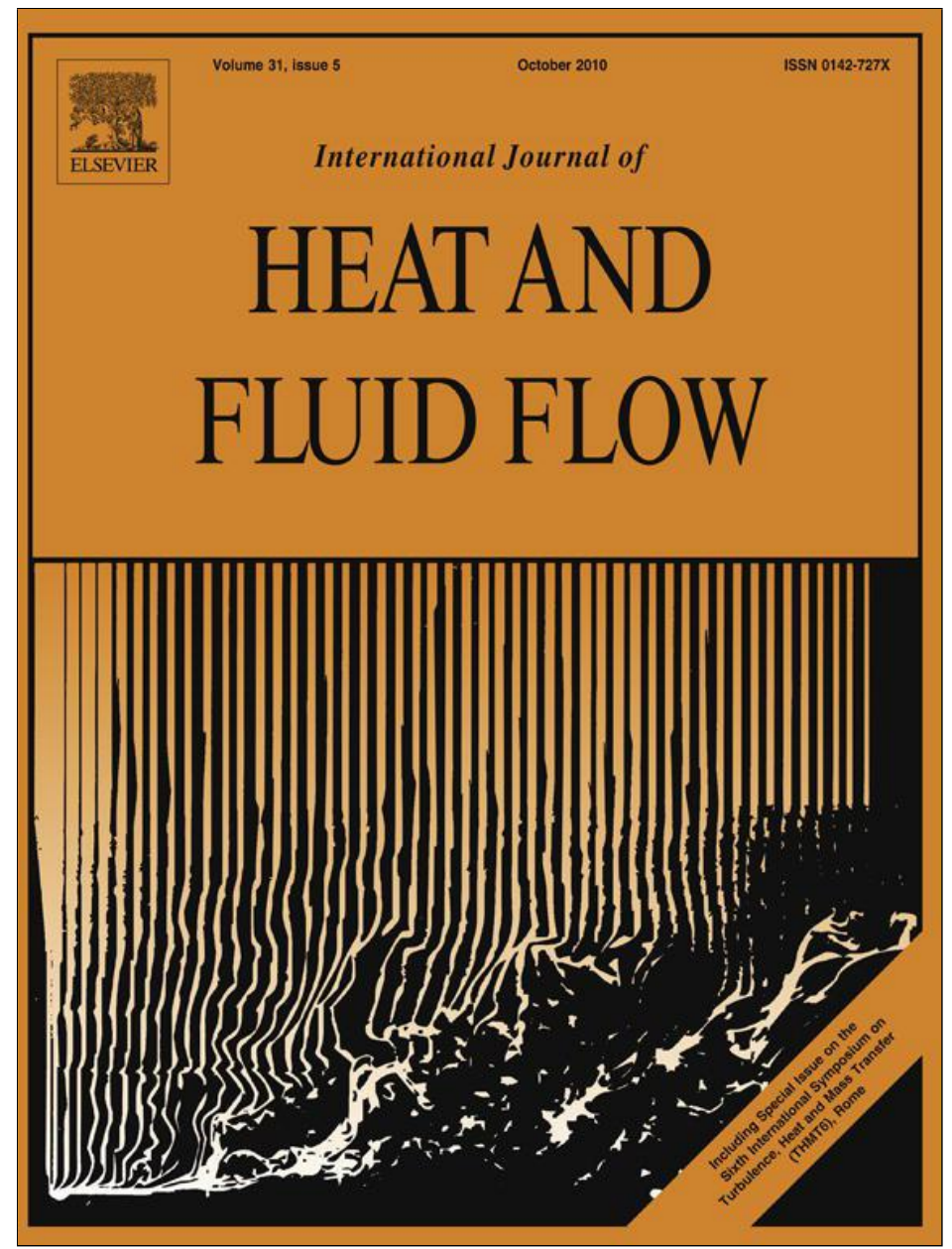

This article appeared in a journal published by Elsevier. The attached copy is furnished to the author for internal non-commercial research and education use, including for instruction at the authors institution and sharing with colleagues.

Other uses, including reproduction and distribution, or selling or licensing copies, or posting to personal, institutional or third party websites are prohibited.

In most cases authors are permitted to post their version of the article (e.g. in Word or Tex form) to their personal website or institutional repository. Authors requiring further information regarding Elsevier's archiving and manuscript policies are encouraged to visit:

http://www.elsevier.com/copyright 


\title{
The distribution of wall shear stress downstream of a change in roughness
}

\author{
J.B.R. Loureiro ${ }^{\mathrm{a}, *}$, F.B.C.C. Sousa ${ }^{\mathrm{b}}$, J.L.Z. Zotin ${ }^{\mathrm{a}}$, A.P. Silva Freire ${ }^{\mathrm{b}}$ \\ ${ }^{a}$ Scientific Division, Brazilian National Institute of Metrology (DINAM/DIMCI/INMETRO), 22050-050 Rio de Janeiro, Brazil \\ ${ }^{\mathrm{b}}$ Mechanical Engineering Program (PEM/COPPE/UFRJ), C.P. 68503, 21941-972 Rio de Janeiro, Brazil
}

\section{A R T I C L E I N F O}

\section{Article history:}

Received 30 November 2009

Received in revised form 14 June 2010

Accepted 19 June 2010

Available online 14 July 2010

\section{Keywords:}

Wall shear stress

Roughness

Turbulence

Laser-Doppler anemometry

\begin{abstract}
A B S T R A C T
In the present work, six different experimental techniques are used to characterize the non-equilibrium flow downstream of a rough-to-smooth step change in surface roughness. Over the rough surface, wall shear stress results obtained through the form drag and the Reynolds stress methods are shown to be mutually consistent. Over the smooth surface, reference wall shear stress data is obtained through two optical methods: linear velocity profiles obtained through laser-Doppler anemometry and a sensor surface, the diverging fringe Doppler sensor. The work shows that the two most commonly used methods to determine the wall shear stress, the log-law gradient method and the Reynolds shear stress method, are completely inappropriate in the developing flow region. Preston tubes, on the other hand, are shown to perform well in the region of a non-equilibrium flow.
\end{abstract}

(c) 2010 Elsevier Inc. All rights reserved.

\section{Introduction}

The wall shear stress is a parameter of paramount importance in the characterization of the morphological structure of attached turbulent boundary layers. Irrespective of the type of surface, whether rough or smooth, the characteristic velocity and length scales in the inner flow regions are always defined in terms of the wall shear stress.

Unfortunately, many methods used to evaluate the wall shear stress rely on the hypothesis that at least part the boundary layer is in an equilibrium state. Hence, in situations where this condition is not followed exactly, all fundamental premises that have led to the development of a particular method need to be carefully considered.

A problem of traditional interest in many areas of application is that of a turbulent boundary layer subjected to a sudden change in surface roughness. The inherent non-equilibrium condition of the wall region for this type of flow naturally implies that any consistency between wall shear stress estimations is very difficult to achieve. In fact, Antonia and Luxton (1972) showed that skin-friction results obtained from the slope of mean velocity semi-log plots were much lower than those obtained with a Preston tube.

The present work studies in great detail the near wall region of the flow field downstream of a rough-to-smooth sudden change in roughness. In particular, the wall shear stress $\left(\tau_{w}\right)$ is evaluated through six different techniques so that reliable data can be indubitably established. The difficulties encountered by other authors

\footnotetext{
* Corresponding author.

E-mail address: jbrloureiro@gmail.com (J.B.R. Loureiro).
}

to obtain accurate measurements of $\tau_{w}$ on the smooth wall are circumvented by two of the techniques; they are based on optical methods and can characterize the flow to within $100 \mu \mathrm{m}$ of the wall.

In the experiments, $\tau_{w}$ is evaluated from extrapolation of measurements of the Reynolds shear stress, from the slope of the logarithmic velocity distribution in the fully turbulent region, from the slope of the linear velocity distribution in the viscous region, from Preston tube readings, from a MEMS-based optical microsensor, and, in the case of the rough surface, from drag estimations obtained by tapping the roughness elements. The reference data furnished by the two optical methods are here used to critically assess the other techniques.

The downstream fetch required before the flow achieves equilibrium condition is characterized by mean velocity and turbulence profiles obtained through two-channel laser-Doppler anemometry (LDA) and two-dimensional particle image velocimetry (PIV). The existence of a "virtual" zero-plane of displacement ${ }^{1}$ downstream of the roughness change is discussed. This is a concept that needs much discussion in a non-equilibrium region following a rough-tosmooth surface change (Malhi, 1996). In fact, even the estimation of the wall shear stress through consideration of the existence of a region adjacent to the wall in which the total shear stress is nearly constant needs to be addressed.

In regions where roughness alternate, a flowing fluid does not immediately adapt at all heights to the local wall conditions, but does so in a layer adjacent to the wall normally referred to as the

\footnotetext{
1 The distance from the bottom of the roughness elements to which a Clauser chart must be referred to.
} 
internal boundary layer (IBL) (Blom and Wartena, 1969). Above this layer, the flow is characteristic of the upstream conditions except for changes resulting from a flow acceleration-term and a term representing the velocity change due to the displacement of the streamlines (Townsend, 1965). The development of the internal boundary layer with downstream distance was early studied by Blom and Wartena (1969), who showed that while velocity profiles evaluated from previously advanced theories (Elliott, 1958; Panofsky and Townsend, 1964 and Townsend, 1965) agree well with experiments, predictions for the wall shear stress disagree strongly. Much of the early work on flow over changing terrain is reviewed by Smits and Wood (1985) and Garratt (1990). In addition to an analysis of the available experimental data, the boundary layer structure and calculation methods are discussed.

A recent contribution to experimental study on roughness change is the work of Cheng and Castro (2002). Their particular concern was to characterize the roughness sublayer. The common expectation is that downstream of a step change with a substantial increase in roughness, there exists a sublayer - the lowest $10 \%$ of the IBL - in which (i) a constant shear stress layer is formed in equilibrium with the new roughness and (ii) the mean velocity is characterised by a logarithmic profile (Kaimal and Finnigan, 1994). In this region, the inertial sublayer (IS), the flow is horizontally homogeneous and similarity theories are applicable. Below the IS, there is a region - the roughness sublayer (RS) - where the geometry of the roughness strongly influences the flow properties. In this region, the existing inhomogeneities make it very difficult to interpret the flow statistics, including the wall shear stress. Cheng and Castro (2002) identify the downstream fetch needed before flow similarity is achieved and a logarithmic region can be observed. The downstream distance the growing equilibrium layer required to encompass the RS and develop the IS was estimated to be $160 z_{02}\left(z_{02}=\right.$ downstream roughness height).

When changes occur from a rough to a smooth surface, the picture seems different. While the wall shear stress adjusts immediately to the new boundary condition, the shear stress in the fully turbulent region adjusts slowly. For this reason, wall shear stress calculations $\left(\tau_{w}\right)$ based on the slope of semi-logarithmic graphs and on measurements of the Reynolds shear stress must be considered with caution. An accurate determination of $\tau_{w}$ for flows over rough walls depends critically on an estimation of the zero-plane displacement $(d)$. Hence, since the readjustment of the internal sublayer to the smooth wall conditions is slow, a residual $d$ must be considered in the calculations of $\tau_{w}$. In previous studies of rough-to-smooth step changes, notably Antonia and Luxton (1972), this procedure, if any, is not well described. Here we show that even with this particular concern, wall shear stress predictions based on Preston tube readings provide much more consistent results than predictions based on both the velocity gradient and the Reynolds shear stress methods.

\section{Theoretical background to the measurement techniques}

\subsection{The velocity gradient method in the viscous region}

In theory, the simplest way to determine $\tau_{w}$ for a Newtonian fluid is to make

$\tau_{w}=\mu \frac{d u}{d z}, \quad$ at $z=0$

The practical difficulty with Eq. (1) is that for most flows the viscous sublayer is very thin - of the order of $v / u_{*}, u_{*}=$ friction velocity - so that $d u / d z$ has to be estimated from measurements made very close to the wall, typically at distances of less than $5 v / u_{*}$.

\subsection{The Reynolds shear stress method}

Outside the viscous layer, the Reynolds shear stress is the dominant part of the total shear stress. Then, by introducing the hypothesis that in the neighborhood of the wall the shear stress remains constant, we have

$\tau_{w}=-\rho \overline{u^{\prime} w^{\prime}}$.

\subsection{The velocity gradient method in the fully turbulent region}

The gradient method developed by Clauser (1954) to determine the skin-friction relies on the existence of self-preservation, and, in particular, on the universality of the parameters appearing in the law of the wall. For a rough surface, this method has been modified by Perry and Joubert (1963) and by Perry et al. (1969) to account for the zero-plane displacement, $d$, and the roughness height, $z_{0}$.

For a rough surface, it is possible to write

$\frac{u}{u_{*}}=\frac{1}{x} \ln \left(\frac{z-d}{z_{0}}\right)$

where, $u_{*}$ is the friction velocity $\left(=\sqrt{\left(\tau_{w} / \rho\right)}\right), x=0.4, d$ is the distance from the bottom of the roughness elements to which the eddy diffusivity extrapolates to zero and $z_{0}$ is a parameter characteristic of the roughness, the roughness height.

As can be seen from Eq. (3), a plot of $u$ against $\ln (z-d)$ in a region of equilibrium flow should furnish a straight line whose slope corresponds to the value of $u_{*}$. A major difficulty however arises from the fact that $d$ is not known a priori. Many approximate methods have been proposed in literature to determine the position of the plane of displacement, $d$. Here, to search for values of $u_{*}$ and $z_{0}$, global optimization algorithms are used. These algorithms attempt to find the global optimum by allowing decrease as well as increase of the objective/merit function. Usually they are computationally expensive. Four different methods were applied to Eq. (3) for solution search: Nelder-Mead method, differential evolution, simulated annealing and random search. Only when all four methods furnish consistent results, with accuracy down to the sixth decimal fraction, the search is stopped.

For flow over a smooth wall, $d$ is identical to zero and $z_{0}=(v)$ $\left.u_{*}\right) e^{-\varkappa A}, A=5.0$.

\subsection{Preston tube}

The principle of operation of a Preston tube is very simple since it relies solely on the difference between the pressure recorded by a Pitot tube resting on a surface and the undisturbed static pressure. The underlying idea is that there exists a near wall region whose velocity distribution is mutual to boundary layers and pipe flows and is determined by the surface shear stress. This assumption gives us the means of determining the friction velocity $\left(u_{*}\right)$ from calibration curves obtained in fully developed pipe flow.

Some early criticism regarding the soundness of the method of Preston to measure $u_{*}$ was convincingly refuted by Head and Rechenberg (1962) and Patel (1965) through experiments conducted in strong favorable and adverse pressure gradients. In very detailed experiments, Patel (1965) established definitive calibration curves, valid in well defined intervals expressed in terms of $u_{*} D / 2 v$, where $D$ is the external tube diameter.

In the present data reduction, the surface Pitot tube had external and internal diameters of respectively 3.0 and $2.6 \mathrm{~mm}$. The calibration, in the range $1.5<\log \left(\tau_{w} D^{2} / 4 \rho v^{2}\right)<3.5$, was used according to the empirical relation (Patel, 1965):

$y^{*}=0.8287-0.1381 x^{*}+0.1437\left(x^{*}\right)^{2}-0.0060\left(x^{*}\right)^{3}$, 
where $x^{*}=\log \left(\Delta p_{p} D^{2} / 4 \rho v^{2}\right), y^{*}=\log \left(\tau_{w} D^{2} / 4 \rho v^{2}\right)$ and $\Delta p_{p}$ is the difference between the Pitot and static pressure.

To show the consistency with his analysis, Preston obtained successfully the constants occurring in the law of the wall from the Preston tube calibration curves.

\subsection{Optical microsensor}

The diverging fringe Doppler sensor is a MEMS-based optical sensor designed to measure the flow velocity gradient at 75$105 \mu \mathrm{m}$ above the sensor surface (Gharib et al., 2002; Fourguette et al., 2004). The small dimensions of this sensor make it an ideal instrument to measure $\tau_{w}$ in the adjustment flow region of a rough-to-smooth surface change.

The system is comprised of a Micro-S sensor, a sensor driver unit that contains the laser source, and a burst processor, responsible for band-pass filtering and conditioning the raw signal that is digitized through a National Instruments board at the computer. The signal processing is based on a FFT analysis that provides time resolved shear stress measurements and associated statistics.

The Micro-S sensor has body dimensions of $6 \mathrm{~mm}$ diameter and $30 \mathrm{~mm}$ length and has to be placed flush to the wall with an acceptable uncertainty of $\pm 5 \mu \mathrm{m}$. A laser diode of $15 \mathrm{~mW}$ and $659 \mathrm{~nm}$ propagates through a fiber optic cable to the probe's head, where a diverging fringe pattern is formed. The diverging interference fringes originate at the surface and extend directly into the flow, as illustrated in Fig. 1. Typical dimensions of the measurement volume are $30 \mu \mathrm{m} \times 30 \mu \mathrm{m} \times 15 \mu \mathrm{m}$. The working distance from the wall is $100 \mu \mathrm{m}$ for air flows and the diverging ratio is 0.066 . This technique can provide wall shear stress measurements in the range from 0.015 to $140 \mathrm{~Pa}$.

The scattered light from the particles passing through the fringes is collected by a window at the surface of the sensor. The measurement volume is defined by the intersection of the transmitter and receiver fields. The seeding particles that cross the measurement volume create frequency modulated bursts, similar to LDA characteristic signals, except that the Doppler frequency is

(a)
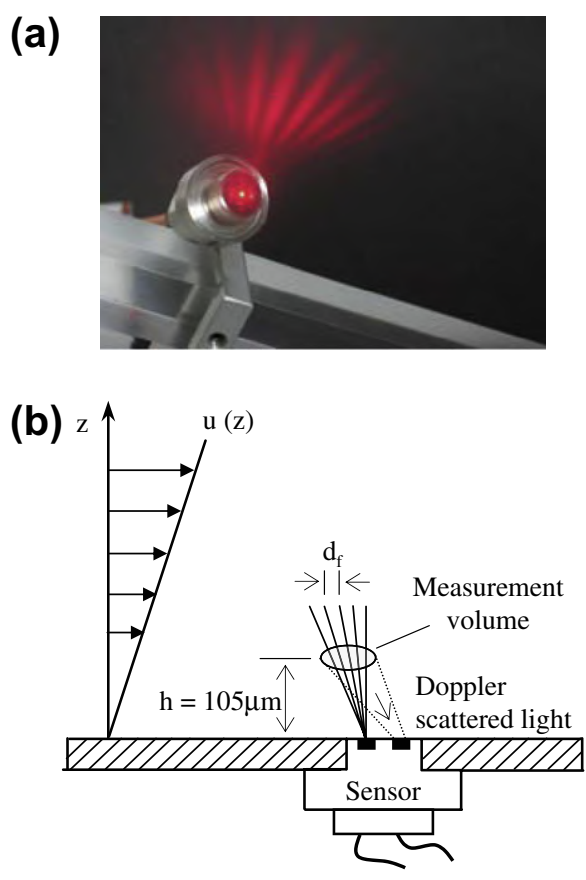

Fig. 1. Laser-based optical microsensor for shear stress measurements: (a) illustration of the probe, (b) schematic diagram of the principles of operation. now proportional to the velocity gradient at the immediate vicinity of the wall. Considering the asymptotic structure of the classical boundary layer, the velocity distribution along the viscous sublayer is known to be linear. As a consequence, once measurements are performed inside this thin region, the velocity gradient at the wall can be calculated straightforwardly from just one point of measured velocity, as long as its distance from the wall is accurately known. This simple concept summarizes the principle of operation of the Micro-S sensor.

In analogy to laser-Doppler anemometry, the measured velocity is linearly dependent on the distance between the fringes, $d_{f}$, and on the Doppler frequency $f_{D}$, i.e. $u=d_{f} f_{D}$. Given the diverging character of the interference fringe pattern, the distance between the fringes is a function of the diverging rate, $k$, and the distance of the measurement volume from the wall, $h$, so that $d_{f}=k h$. From the definition the wall shear stress $\left(\tau_{w}=\mu \partial u / \partial z\right)$ we have $\tau_{w}=\mu d_{f}$ $f_{D} / h$, which leads to the direct linear relation between the wall shear stress and the Doppler frequency, i.e. $\tau_{w}=\mu k f_{D}$.

The major assets of this technique is that it is non-intrusive, does not require calibration, has a linear response and can be used in any flow problem as long as a viscous sublayer can be identified.

\subsection{Form drag}

A removable roughness element fitted with pressure taps can be used to determine the wall shear stress. By defining a control volume around this element, a simple $x$-momentum balance (Perry et al., 1969) gives

$\frac{\tau_{w}}{\frac{1}{2} \rho U_{e}^{2}}=\frac{K}{\lambda}\left(C_{D}-\frac{\Delta P_{L}}{\frac{1}{2} \rho U_{e}^{2}}\right)$,

where $C_{D}$ is the drag coefficient of the roughness element, $\Delta P_{L}$ is the free stream pressure difference, $U_{e}$ is the external flow velocity and $K$ and $\lambda$ are the height and pitch of the roughness.

\section{Experiments}

The experiments were carried out in the low-turbulence wind tunnel of the Laboratory of Turbulence Mechanics (PEM/COPPE/ UFRJ). The tunnel is an open circuit tunnel with a test section of dimensions $300 \mathrm{~mm} \times 300 \mathrm{~mm} \times 8000 \mathrm{~mm}$. The roughness elements were fitted into the first four meters. The remaining four meters were fitted with an acrylic surface. A general view of the wind tunnel is shown in Fig. 2.

The rough surface was a transversely grooved surface constructed with rectangular aluminium bars of 6.0 by $4.8 \mathrm{~mm}$ rectangular cross section (see Fig. 3). Every roughness element was made removable so that another element constructed with pressure taps could be fitted anywhere into the roughness pattern. The pressure taps, twenty six in all, were drilled directly onto the removable element at varying distances from the wall. The pressure tubes were connected directly to a Furness micromanometer, enabling pressure differences to be measured to up an accuracy of $0.001 \mathrm{~Pa}$.

Measurements were performed for values of the free stream velocity of $8 \mathrm{~ms}^{-1}$; the free stream-level of turbulence was about $0.2 \%$. Mean velocity and turbulence statistic data were obtained with a two component laser-Doppler anemometer (LDA) and a two-dimensional particle image velocimeter (PIV).

The two-component Dantec laser-Doppler anemometry system used a $400 \mathrm{~mW}$ Ar-ion tube laser and was operated in the backscatter mode to measure mean and fluctuating velocity fields. A Bragg cell unit was used to introduce a digitally-controlled electronic shift in order to resolve the direction of the flow field and give correct measurements of near-zero mean velocities. The beams were oriented in space such that the green beams measured 


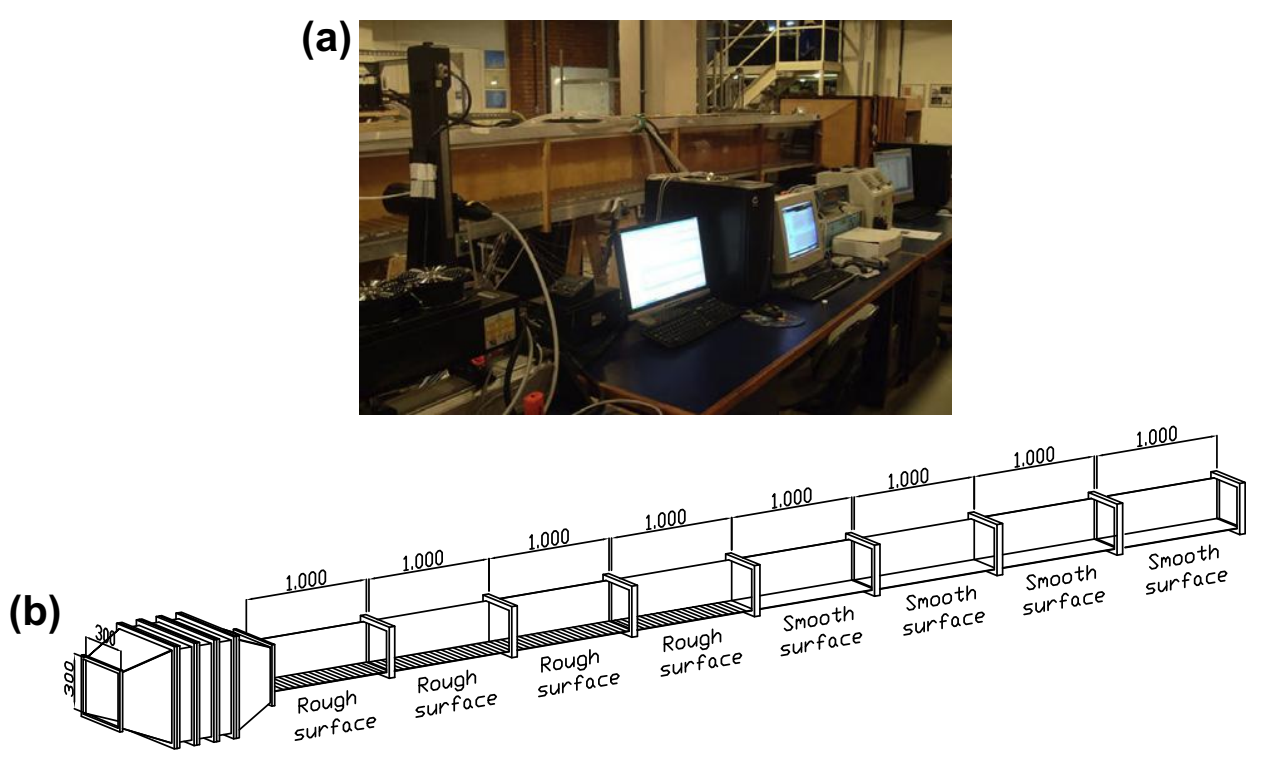

Fig. 2. Wind tunnel: (a) general view, (b) schematic diagram.

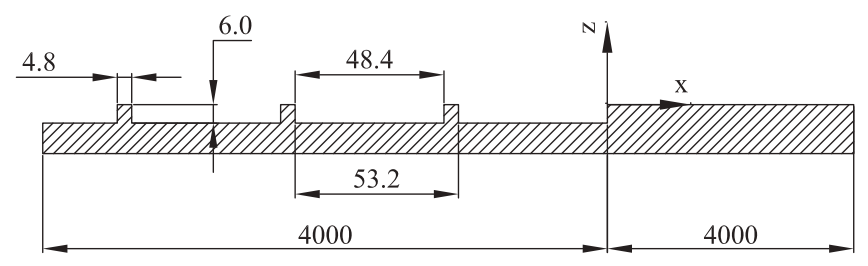

Fig. 3. Geometry of roughness elements. Dimensions are in mm. Note the position of the coordinate system.

the wall-parallel velocity component, $u$, and the blue beams measured the wall-normal component, $w$. The four light beams that emerged from the $60 \mathrm{~mm}$ diameter FiberFlow probe were made to pass through a beam translator and a beam expander with expansion ratio of 1.98. These optical components were used to increase the beam spacing and, as a consequence, to provide a smaller measurement volume with higher laser power, maximizing the signal-to-noise ratio. A careful alignment procedure of these optical components were made with the aid of a microscope lens to assure that the four light beams were all crossing precisely at the same point in space where the receiving optical fiber is focused.

Front lens with $310 \mathrm{~mm}$ focus length were mounted on the probe to accurately position the measurement volume on the centerline of the wind tunnel. Before being collected by the photomultipliers, the scattered light was made to pass through interference filters of $514.5 \mathrm{~nm}$ and $488 \mathrm{~nm}$, so that only the green and blue lights were received on each photomultiplier, respectively. Table 1 lists the main characteristics of the laser-Doppler system used. The signals from the photomultipliers were digitized and processed through a burst spectrum analyzer BSA P60 operating in single measurement per burst mode. The Dantec BSA Flow Software 4.50 were used to calculate the Doppler frequencies and the resulting velocity samples. A series of LDA biases were avoided by adjusting the strictest parameters on the data processor and software. The level validation and the signal-to-noise ratio were 8 and 5 respectively. For simultaneous measurements of longitudinal and vertical velocities only overlapped bursts were considered. For the statistics at each point, 50,000 samples were acquired.

In the experiments, the total number of samples for every point of velocity measurement was adjusted to ensure statistical independence between consecutive measurements as well as to furnish
Table 1

Main characteristics of the laser-Doppler system.

\begin{tabular}{ll}
\hline Wavelength & $514.5 \mathrm{~nm}$ (green) \\
Wavelength & $488 \mathrm{~nm}$ (blue) \\
Half-angle between beams & $1.604^{\circ}$ \\
Fringe spacing & $2.261 \mu \mathrm{m}$ (green) \\
Fringe spacing & $2.145 \mu \mathrm{m}$ (blue) \\
Beam spacing & $71 \mathrm{~mm}$ \\
Beam diameter & $4.1 \mathrm{~mm}$ \\
Dimensions of the measur. volume & \\
Major axis & $435.3 \mu \mathrm{m}$ (green) \\
Major axis & $412.9 \mu \mathrm{m}$ (blue) \\
Minor axis & $49.53 \mu \mathrm{m}$ (green) \\
Minor axis & $46.98 \mu \mathrm{m}$ (blue) \\
\hline
\end{tabular}

an interval of confidence of 95\%. The random uncertainty was calculated according to ANSI/ASME Standards using a t-distribution. Note that turbulent quantities do not often follow a Gaussian distribution, so that usual simplifications can lead to significant errors if normality is not previously verified. The systematic uncertainty was assessed in terms of inaccuracies on the measurement of the intersection angle and on the correct alignment of the probe in relation to the flow. Velocity bias was avoided with the use of transit time weighting. The overall uncertainty was calculated as described in ANSI/ASME (1986) and expressed in percentages of the free stream velocity, $U_{e}$ (for the mean velocities) and the square of the friction velocity, ( $\left.u_{*}^{2}\right)$ (for the Reynolds stress components). The main reference to the present uncertainty calculations is Coleman and Steele (1999).

Typical uncertainties associated with the mean velocity data $U, W$ - are below $0.2 \% U_{e}$. Regarding the Reynolds stress components, uncertainties were estimated to be $2.3 \% u^{2}$.

The PIV measurements were performed with a two-dimensional Dantec system. The light source was furnished by a double pulsed Nd:YAG laser that produced short duration (10 ns) high energy $(120 \mathrm{~mJ})$ pulses of green light $(532 \mathrm{~nm})$. The collimated laser beam was transmitted through a cylindrical $(15 \mathrm{~mm})$ and a spherical $(500 \mathrm{~mm})$ lens to generate a $1 \mathrm{~mm}$ thick lightsheet. The reflected light was recorded at $5 \mathrm{~Hz}$ by a CCD camera with $1280 \times 1024$ pixels and 12-bit resolution. The cameras were fitted with a Nikkor $105 \mathrm{~mm} \mathrm{f} / 2.8 \mathrm{D}$ lenses. The air was seeded with a Laskin nozzle. Image calibration was made by taking pictures of a reference target specially designed for the present purpose. 
For all the measurements, computational conditions for the velocity vectors were fixed. Adaptive correlation (Dynamic Studio Software) has been processed on $32 \times 32$ pixels-size final interrogation windows, with $50 \%$ overlap, which gives $64 \times 64$ vectors. The pixel resolution is $6.45 \times 6.45 \mu \mathrm{m}$. Particle image treatment using subpixel cell shifting and deformation allowed bias and random error reduction. A widely accepted estimation of the absolute displacement error using these algorithms is 0.05 pixels. Different thresholds including signal-to-noise ratio and velocity vector magnitude were used as post-processing steps.

The two measurement techniques used in the present work LDA and PIV - rely on the presence of small seed particles suspended in the flow field. Ideally, the seeding used for laser-based measurements must be small and light enough to follow the fluctuations of the mean flow stream ensuring an adequate aerodynamic response to velocity gradients and turbulence intensities. A compromise between reducing the particle size to improve the flow tracking and increasing the particle volume to improve light scattering and maximizing the signal-to-noise ratio is therefore a very difficult task (Durst et al., 1981). However, since the accuracy of the velocity field determination is ultimately limited by the ability of the scattering particles to follow the instantaneous flow field, a careful choice of the seed particles and a thorough investigation to assure that the seed injection is not affecting the flow field of interest is a time consuming but necessary step of the experimental set up procedure.

In the present work, seeding investigations have used two different types of flow tracers - a fog generator and a Laskin nozzle - and a variety of injection points have been tested. Both flow generators were filled with the same aqueous solution of dialcohol-glycerol mixture. The typical diameter of particles furnished by the fog generator is about $1 \mu \mathrm{m}$. The major limitations of fog generators are the intermittent discharge of particles, the high density of the particle flow and the eventual contamination of the surrounding environment by the fog. Droplets produced by the atomizer (Laskin nozzle) vary in size between the range of $0.5 \mu \mathrm{m}$ and $5.0 \mu \mathrm{m}$, with the particle diameter being a function of the pressure of the compressed air feeding line and the working fluid. For the present work, a 5 bar pressure input provided particles of approximately $3 \mu \mathrm{m}$ in size. Under these conditions, the Laskin nozzle provided a homogeneous and continuous flow of tracer particles, solving the major problems observed with the use of the fog generator.

For both the LDA and PIV techniques the Laskin nozzle provided the best results, assuring a continuously seeded flow with particles that are able to follow the flow field fluctuations but yet scatter enough light to provide a good signal-to-noise ratio. The investigation for the most appropriate injection position showed that connecting the tracer particles directly to the inlet of the fan furnished the most appropriate homogeneous distribution of the seeds in flow field rather then injecting the tracer particles immediately downstream the contraction of the wind tunnel or directly in the test section itself.

\section{Results}

The general flow pattern that is formed in the first cavity before the smooth surface is reached is shown in Fig. 4 as given by the PIV measurements. Two large regions of recirculating flow are identified, with the formation of stable vortices. Shedding from the protuberances into the flow is significant. In this case, the external flow after passing over the top of a roughness element penetrates deeply into the cavity. In fact, major disturbances are limited to a distance above the crest of the roughness elements of the order of their height, $K$. These flow features are typical of a ' $K$ '-type surface. In fact, it must be pointed out that the present flow pattern is somewhat different from the flow visualization studies that were

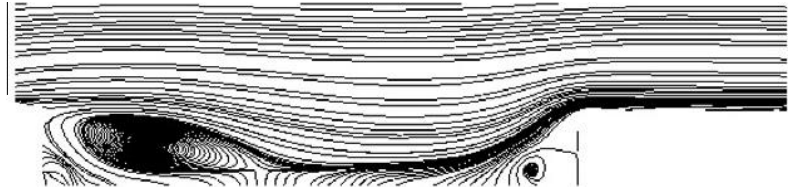

Fig. 4. Flow streamlines between roughness elements. Flow is from left to right

presented in Perry et al. (1969) since two large eddies are apparent. A distinct feature of a ' $K$ ' type roughness is the absence of a stagnation streamline on the leading face of the cavity. The flow is observed to divide around a streamline near the front of the crest. The absence of separated flow on the leading edge of the crest and the presence of trapped stagnant fluid on the trailing side of the cavity are also characteristic of ' $K$ ' type surfaces.

The local mean and turbulent statistics of the flow were assessed through LDA. All results presented in Figs. 5-12 have been obtained with this technique.

The dependence of the flow properties on the location at which measurements are made has been extensively discussed by Cheng and Castro (2002). In the roughness sublayer (RS), the geometry of the roughness strongly influences the flow properties, making it difficult to interpret the flow statistics. Above the RS, there is a region, the inertial sublayer (IS), where flow properties are horizontally homogeneous. The main concern here is to characterize the wall shear stress using measurements taken at the IS. When that is case, the normal practice in literature is to consider measurements in the middle of the roughness elements in the IS region. This practice also allows measurements to be made with less wall interference, particularly if laser-Doppler and hot-wire anemometry techniques are used.

Measured velocity profiles for different positions over the smooth and rough surfaces are shown in Figs. 5 and 6. The linear velocity profiles that hold in the viscous sublayer at positions $x=10,15,20$ and $30 \mathrm{~mm}$ are shown in Fig. 5. In every profile, at least five measurement points have been located in the first $250 \mu \mathrm{m}$. The finite size of the LDA measurement volume naturally introduces an uncertainty regarding the absolute distance from the wall. Here, the wall location has been determined according to the procedure of Durst et al. (1996). The very good discrimination of the linear solution allows Eq. (1) to be used without any discretion for the estimation of $\tau_{w}$.

The velocity profiles in logarithmic coordinates are shown in Fig. 6 . The distortion of the logarithmic velocity profile due to the displacement in origin is apparent from Fig. 6a. The zero-plane displacement, $d$, was estimated through the procedures of Perry and Joubert (1963) and of Perry et al. (1969) and with the search methods previously described: Nelder-Mead, differential evolution, simulated annealing and random search. In these methods, arbitrary values of $d$ are added to the wall distance measured from the bot-

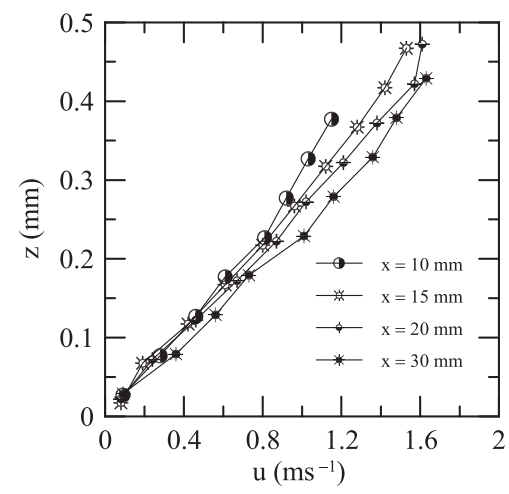

Fig. 5. Linear velocity distributions over the smooth surface. 

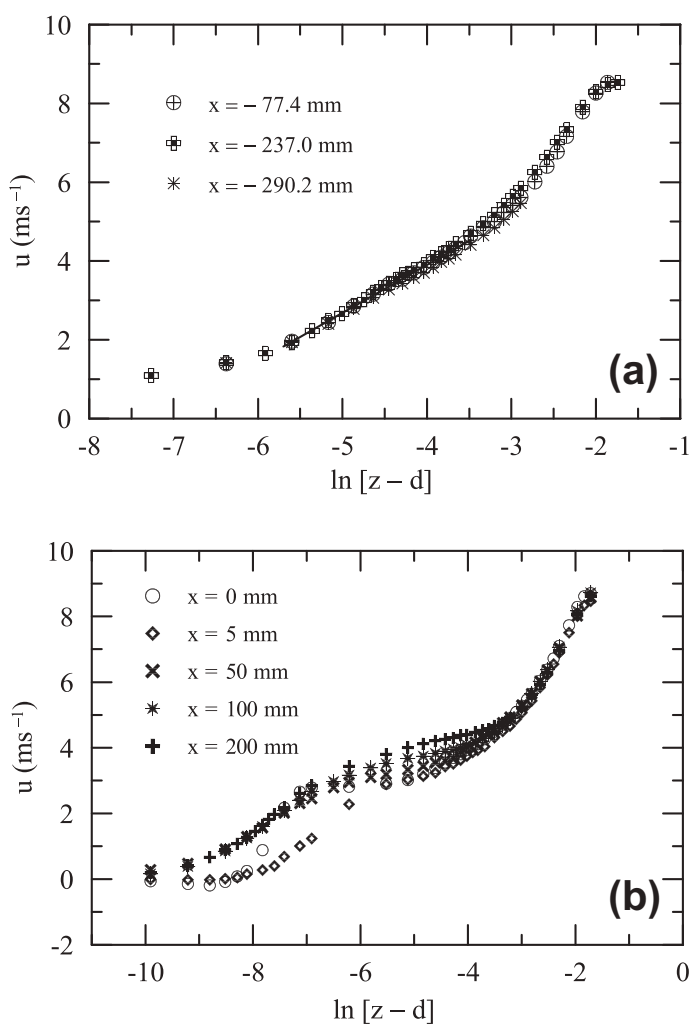

Fig. 6. Velocity profiles over the rough (a) and smooth (b) surfaces.

tom of the roughness elements and a straight line is fitted to the $\log$-law region. The value of $d$ that furnishes the best discriminated logarithmic region is then considered to be the correct value for the zero-plane displacement. The velocity gradient method is illustrated in Fig. 7 for calculations made at position $x=-77.40 \mathrm{~mm}$. For the present flow conditions, $d$ was found to be $4.3 \mathrm{~mm}$.

All three distinct flow regions, the viscous region, the fully turbulent region and the defect region, can be very clearly identified in Fig. 6b. The negative values of velocity in the first points of profiles $x=0$ and $5 \mathrm{~mm}$ indicate the presence of a short separation bubble. Fig. $6 \mathrm{~b}$ shows clearly that on reaching the smooth wall the flow suddenly accelerates, before the boundary layer starts to readjusts to the new surface conditions. The same procedure used above to find $d$ for flow over the rough surface was repeated for the non-equilibrium flow over the smooth wall. At the leading edge of the wall, $x=0, d$ was observed to be different from zero. For all other positions, however, $d$ was found to be virtually zero. In fact,

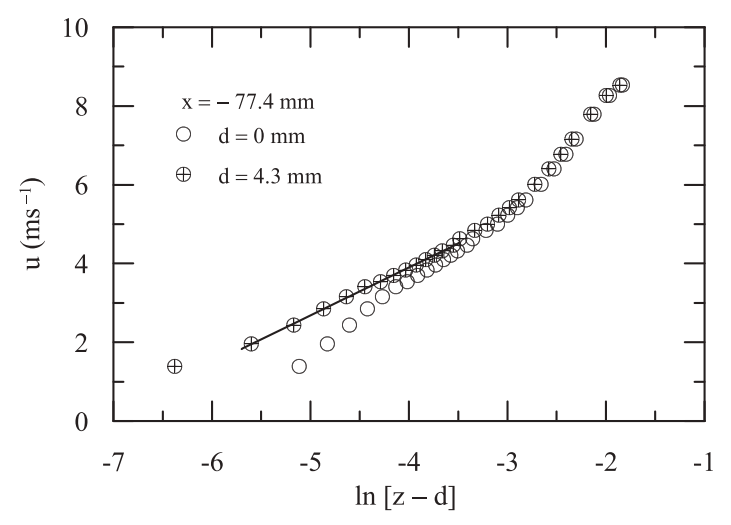

Fig. 7. Illustration of the velocity gradient method.

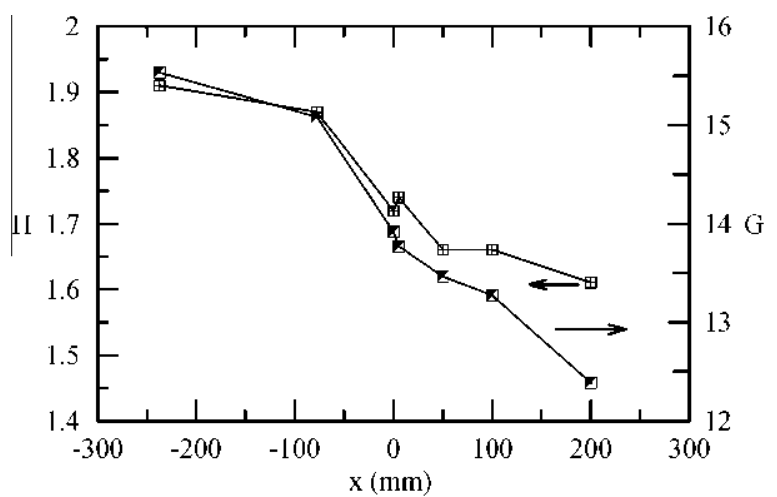

Fig. 8. Variation of the shape $(H)$ and defect profile $(G)$ parameters with $x$.

up to $x=30 \mathrm{~mm}$, measurements were made for every $5 \mathrm{~mm}$ (not shown here). Thus, despite the slow adjustment of some of the flow turbulent statistics - as we shall see - in the fully turbulent region $d$ immediately tends to zero.

The non-equilibrium state of the boundary layer is characterized in Fig. 8, where the shape parameter $H$ and the defect profile parameter of Clauser $G$ are shown. Antonia and Luxton (1972) mention that for flow over a smooth or rough surface self-preservation is achieved for $H$ and $G$ of the order of 1.35 and 6.5 respectively.

These parameters are defined as

$H=\frac{\delta_{1}}{\delta_{2}}, \quad G=\int_{0}^{\infty}\left(\frac{U_{e}-U}{u_{*}}\right)^{2} d\left(\frac{z-d}{\Delta}\right)$,

where $\delta_{1}$ is the boundary layer displacement thickness, $\delta_{2}$ the momentum thickness, $U_{e}$ the external flow velocity and $\Delta=\left(\delta_{1} U_{e}\right)$ $\left.u_{*}\right)$.

The experiments of Antonia and Luxton (1972) give at the last measuring station downstream from the step a value of 1.42 for $H$. At the same station, the experimental values for $G$ are 8.04 and 8.56 (obtained with Preston tube measurements and for two different reference external velocities). The evident conclusion is that their boundary layer is not self-preserving. Here, at the last measuring station, the corresponding values of $H$ and $G$ are 1.61 and 12.39 so that the same conclusion applies.

The distribution of the normal stress $\left(\sigma_{u u}=\sqrt{\overline{{u^{\prime 2}}^{2}}}\right)$ over the rough and smooth surfaces is shown in Fig. 9. Over the rough surface, all longitudinal stress profiles collapse well with each other. A single maximum can be identified at $z \approx 6 \mathrm{~mm}, \sigma_{u u} \approx 1.4 \mathrm{~ms}^{-1}$. Over the smooth surface, however, two local maxima are seen. They are evident at all positions, including $x=0$ and $5 \mathrm{~mm}$. The lower peak is provoked by the large generation of turbulence by the separation bubble at the leading edge, remaining about the same wall distance for all $x$. The higher peak, on the other hand, increases its distance from the wall with increasing $x$. The magnitude of the near wall peak values of $\sigma_{u u}$ for $x$ larger than $50 \mathrm{~mm}$ are lower than their respective values in the rough surface flow. The outer part of the $\sigma_{u u}$-profiles keep their upstream values.

Measurements of the shear stress $\left(\sigma_{u w}=\sqrt{\overline{u^{\prime} w^{\prime}}}\right)$ could not be made too close to the wall due to some geometrical constrains. Over the rough surface, the $\sigma_{u w}$-profiles agree reasonably with each other (Fig. 10a). Over the smooth surface, $\sigma_{u w}$ decreases slightly from the upstream rough-wall values $\left(\approx 0.35 \mathrm{~ms}^{-2}\right)$ for all positions ${ }^{2}$ but $x=200 \mathrm{~mm}$ (Fig. 10b). The slow adjustment of $\sigma_{u w}$ to the new wall conditions is thus very apparent from Fig. 10b.

In turbulent flows, important structural information can be extracted from the higher-order moments. The skewness and flatness

\footnotetext{
${ }^{2}$ Noticeably at $x=50$ and $100 \mathrm{~mm}$.
} 

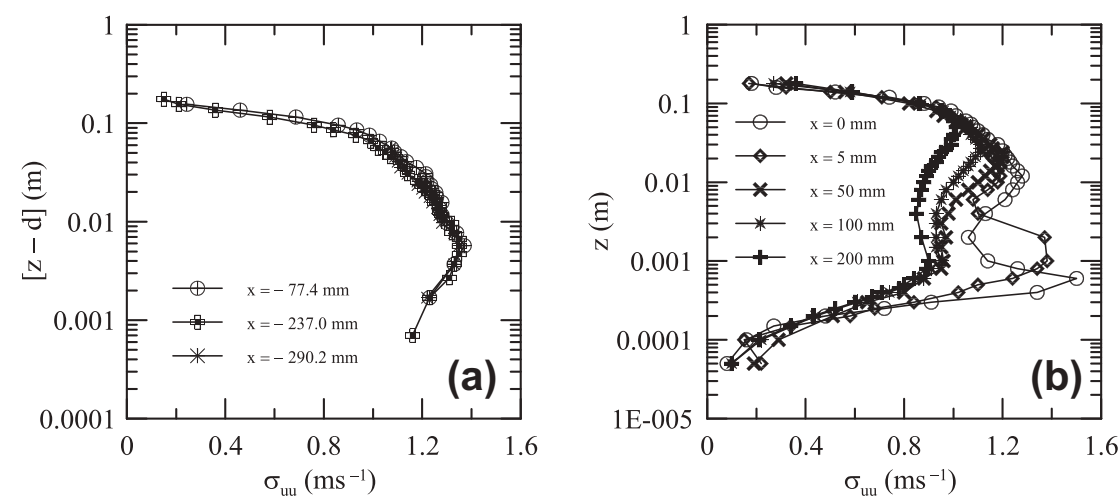

Fig. 9. Longitudinal stress over the rough (a) and smooth (b) surfaces.
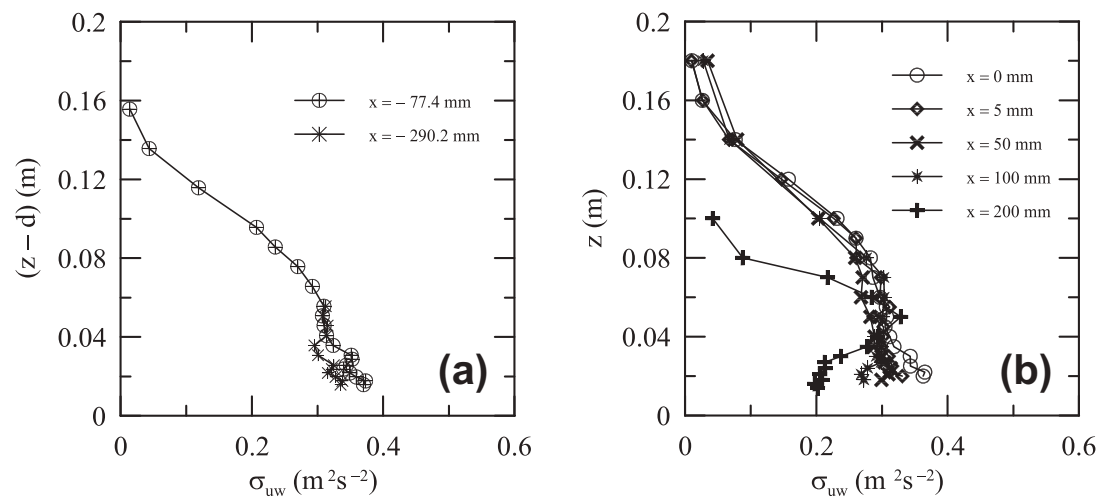

Fig. 10. Reynolds shear stress over the rough (a) and smooth (b) surfaces.

factors for the streamwise velocity fluctuations are defined respectively by

$S_{u}=\overline{u^{\prime 3}} /\left(\overline{u^{\prime 2}}\right)^{3 / 2}$,

$F_{u}=\overline{u^{\prime 4}} /\left(\overline{u^{\prime 2}}\right)^{2}$.

Data with a Gaussian distribution satisfy $S_{u}=0$ and $F_{u}=3$.

The behavior of $S_{u}$ for flow over rough and smooth surfaces is illustrated in Fig. 11. For a smooth wall, $S_{u}$ remains negative across the entire boundary layer (Krogstad and Antonia, 1999; Flack et al., 2007). For a rough wall however, when $z$ is small, positive values of $S_{u}$ are observed, with a peak value of the order of 0.5 . Because the velocity triple products are very sensitive to surface conditions, they can be used to identify the quality of a surface, whether smooth or rough.

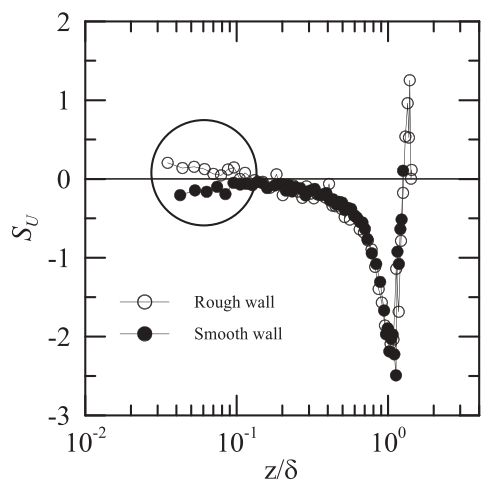

Fig. 11. Near wall skewness behavior for flow over rough and smooth surfaces.
The present measurements over the rough surface give the exact expected trend (Fig. 12a). However, over the smooth surface, in the non-equilibrium region, very large positive values of $S_{u}$ are observed in excess of unity (positions $x=50,100,200 \mathrm{~mm}$; Fig. 12b). The immediate flow adherence to the smooth wall is therefore not followed by an immediate adjustment of $S_{u}$ to its expected negative values. The slow adjustment of the moments of the fluctuating quantities to equilibrium conditions suggests that wall shear stress predictions based on the velocity gradient method and on the Reynolds shear stress method should fail for some distance following the roughness change.

Profiles for the flatness show very high values near the wall (Fig. 12d) and in the outer layer (Fig. 12c and d), where turbulence is highly intermittent. Particularly violent events are noted in the near wall region of the smooth surface. For equilibrium flows, $F_{u}$ is about 2.8 in the fully turbulent region. Here, much higher values of the order of 6,8 are noted.

The values of the friction velocity $\left(u_{*}=\sqrt{\tau_{w} / \rho}\right)$ obtained through the six previously described methods are shown in Fig. 13.

Over the rough surface (negative $x$ ), predictions of $u_{*}$ can be obtained through three methods: (i) the form drag of an individual roughness element (FDM), (ii) the Reynolds shear stress method (RSS) and (iii) the velocity gradient method based on the displacement in height for the turbulent region (VGTR). For large negative positions, results given by the RSS and VGTR agree very well. At position $x=-77.4 \mathrm{~mm}$, estimations based on the FDM and RSS methods provide $u_{*}=0.63$ and $0.59 \mathrm{~ms}^{-1}$ respectively. However, the VGTR underestimates the friction velocity by about $22 \%$, giving $u_{*}=0.46 \mathrm{~ms}^{-1}$. Note that the RSS and VGTR methods can only be used for flows in equilibrium conditions.

Over the smooth wall (positive $x$ 's), five methods can be used to estimate $u_{*}$ : (i) the velocity gradient method (VGM), (ii) RSS, (iii) 

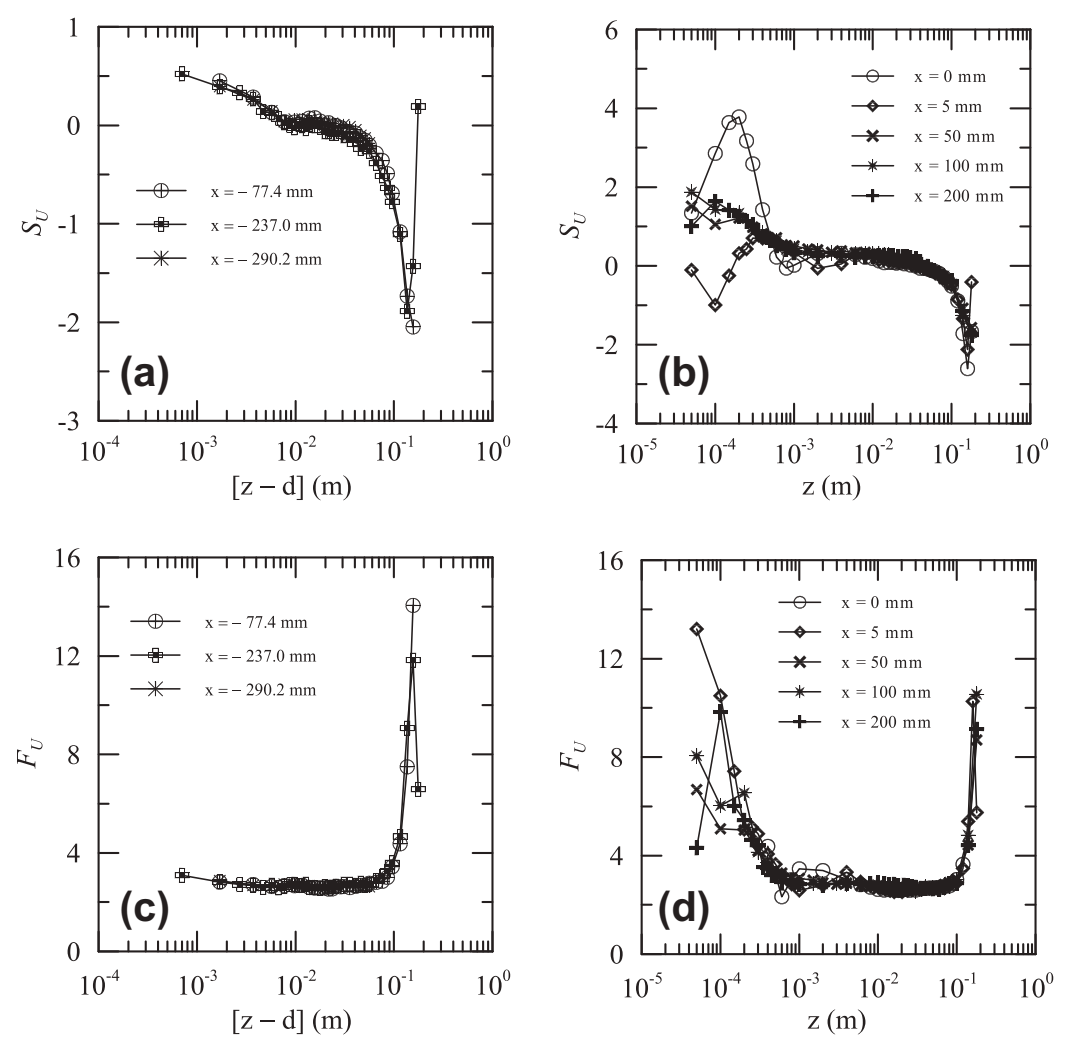

Fig. 12. Turbulence statistics over the rough and smooth surfaces: skewness (a, rough surface; b, smooth surface) and flatness (c, rough surface; d, smooth surface).
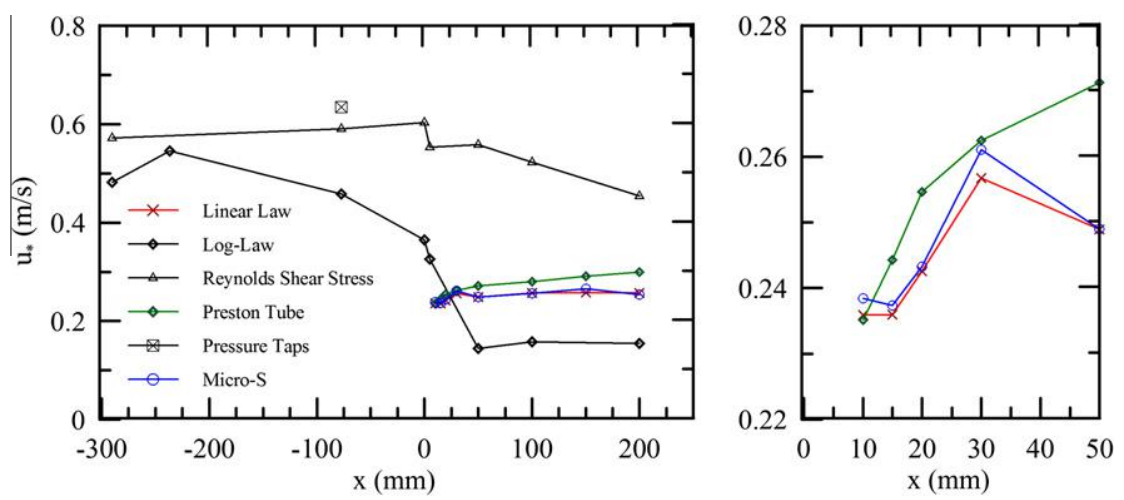

Fig. 13. Skin-friction velocity over the rough and smooth surfaces. The near origin behavior of the VGVR, MS and PT methods are shown on the right.

Micro-S sensor (MS), (iv) the velocity gradient method in the viscous region (VGVR) and (v) the Preston tube (PT). For all eight measuring positions, estimations of $u_{*}$ based on the VGVR, MS and PT methods showed a very good agreement. In fact, both optical methods, VGVR and MS, furnish almost identical results. Estimations by the PT method for small $x$ 's $(<30 \mathrm{~mm})$ coincide almost exactly with the VGVR and MS predictions. For larger $x(>50 \mathrm{~mm})$ the maximum difference is $10.6 \%$ at position $x=200 \mathrm{~mm}$.

As expected, the VGTR and RSS methods at all positive positions do not provide realistic estimations of $u_{*}$. Near $x=0$, these two methods furnish predictions of $u_{*}$ of about $0.34 \mathrm{~ms}^{-1}$ (VGTR) and $0.58 \mathrm{~ms}^{-1}$ (RSS), as compared to the reference value of $0.23 \mathrm{~ms}^{-1}$ (VGVR and MS). At the last measuring station, $x=200 \mathrm{~mm}$, results given by the VGTR and RSS methods are still consistently below $\left(0.15 \mathrm{~ms}^{-1}\right)$ and above $\left(0.45 \mathrm{~ms}^{-1}\right)$ the reference value $\left(0.26 \mathrm{~ms}^{-1}\right)$. This is explained next.

Regarding the estimations given by the RSS method, Fig. 10b shows that $\overline{u^{\prime} w^{\prime}}$ adjusts very slowly to the new wall conditions, thus retaining characteristics that are typical of the upstream surface layer. Therefore, predictions of $u_{*}$ based on $\overline{u^{\prime} w^{\prime}}$ data are not appropriate in the developing region. This fact is very much reflected on the very high predictions shown in Fig. 13. This method must then be ruled out for wall shear stress measurements in regions of roughness change.

Results obtained with the VGTR method are consistently lower than those obtained with the optical methods and the Preston tube. Antonia and Luxton (1972) had previously reported the same conclusion, but with reference measurements provided just by a Preston tube. In fact, Antonia and Luxton (1972) write that "none of the standard smooth wall methods of obtaining skin-friction from mean velocity measurements is reliable for some distance downstream of the roughness change". Their final recommendation is that skinfriction coefficient measurements should be carried out with a floating element balance. Here, we show that, contrary to what is commonly believed, the Preston tube can be used to yield a good estimation of $u_{*}$ in the developing region over the smooth wall. 
The general trend shown in Fig. 13 for the optical and Preston tube estimations is the same as that inferred by Antonia and Luxton (1972). The main concern of the present work has been to investigate the behavior of the wall shear stress in the non-equilibrium region following a rough-to-smooth wall change. Future work will discuss in detail the further downstream region.

\section{Final remarks}

The present work has critically analyzed six different methods for the estimation of the wall shear stress in regions of roughness change. In particular, the near wall region following a rough-tosmooth change has been investigated in great detail.

Over the rough surface, three methods were used: the log-law gradient method, the Reynolds stress method and the form drag method. Far upstream from the point of change in roughness $(x=0)$ the first two methods gave consistent results. Approaching to $x=0$, the log-law method deviated strongly from the other predictions.

Over the smooth surface, five methods were used: the log-law gradient method, the linear-law gradient method, the Reynolds shear stress method, the Preston tube method and the Micro-S sensor method. The two most commonly used methods, the log-law gradient method and the Reynolds shear stress method, are shown to be completely inappropriate to estimate the wall shear stress in the developing region. Respectively, they give results systematically much lower and much higher than those provided by the optical methods.

The Preston tube, on the other hand, proved to be a useful instrument to estimate $u_{*}$. This instrument is known to depend critically on calibration constants that rely on the universality of the law of the wall, specifically on $x$ and $A$. Here, we used tubes with external $(3.0 \mathrm{~mm})$ and internal $(2.6 \mathrm{~mm})$ diameters that were shown to perform well with Patel's calibration curves (Eq. (4)).

The Reynolds shear stress, the velocity gradient and the Preston tube methods consider that close to the wall in the turbulent shear flow there is a region in which the flow is primarily determined by the wall shear stress. In other words, they rely on the existence of the law of the wall. The first two methods, however, are based on estimates obtained from local data. The Preston tube readings correspond to pressure data averaged over the entire tube diameter. This integral character of the Preston tube appears to make it less sensitive to measurements off equilibrium condition.

Determining the wall shear stress has always been a difficult problem that has plagued many authors. In developing, non-equilibrium flows, this problem is aggravated manifold. In this work, reliable results for skin-friction for flow over a rough-to-smooth surface change have been obtained.

\section{Acknowledgements}

F.B.C.C. Sousa benefited from a Research Scholarship from the Brazilian Ministry of Education through Capes. J.L.Z. Zotin is reci- pient of a Research Fellowship from the Rio de Janeiro Research Foundation (FAPERJ). A.P. Silva Freire is grateful to the Brazilian National Research Council (CNPq) for the award of a Research Fellowship (Grant No. 303982/2009-8). The work was financially supported by the Brazilian National Research Council (CNPq) through Grant No. 473588/2009-9 and by the Rio de Janeiro Research Foundation (FAPERJ) through Grant No. E-26/170.005/ 2008. J.B.R. Loureiro is thankful to the Brazilian National Research Council (CNPq) for the financial support to this research through Grant 475759/2009-5.

\section{References}

American National Standards Institute/American Society of Mechanical Engineers, 1986. Measurement uncertainty, PTC 19.1.

Antonia, R.A., Luxton, R.E., 1972. The response of a turbulent boundary layer to a step change in surface roughness. Part 2. Rough to smooth. J. Fluid Mech. 53, 737-757.

Blom, J., Wartena, L., 1969. The influence of changes in surface roughness on the development of the turbulent boundary layer in the lower layers of the atmosphere. J. Atmos. Sci. 16, 255-265.

Cheng, H., Castro, I.P., 2002. Near-wall flow development after a step change in surface roughness. Boundary-Layer Meteorol. 105, 411-432.

Clauser, F.H., 1954. Turbulent boundary layers in adverse pressure gradients. J. Aeronaut. Sci. 21, 91-108.

Coleman, H.W., Steele, W.G., 1999. Experimentation and Uncertainty Analysis for Engineers. John Wiley \& Sons. pp. 275.

Durst, F., Melling, A., Whitelaw, J.H., 1981. Principles and Practice of Laser-Doppler Anemometry. Academic Press, London.

Durst, F., Kikura, H., Lekakis, I., Jovanovic, J., Ye, Q., 1996. Wall shear stress determination from near-wall mean velocity data in turbulent pipe and channel flows. Exp. Fluids 20, 417-428.

Elliott, W.P., 1958. The growth of the atmospheric internal boundary layer. Trans. Am. Geophys. Union 39, 1048-1054.

Flack, K.A., Shultz, M.P., Connely, J.S., 2007. Examination of a critical roughness height for outer layer similarity. Phys. Fluids 19, 95-104.

Fourguette, D., Gonzalez, P., Modarress, D., Arik, E., Wilson, D., Koochesfahani, M., 2004. Optical measurement of wall shear stress with emphasis on flows near separation. In: 24th AIAA Aerodyn. Measur. Tech. and Ground Testing Conference, Portland, Oregon.

Garratt, J.R., 1990. The internal boundary layer - a review. Boundary-Layer Meteorol. 38, 369-394.

Gharib, D., Modaress, M., Fourguette, D., Wilson, D., 2002. Optical microsensors for fluid flow diagnostics. In: 40th AIAA Aeros. Sci. Meeting and Exhibition, Reno, NV.

Head, M.R., Rechenberg, I., 1962. Preston tube as a means of measuring skin friction. J. Fluid Mech. 14, 1-17.

Kaimal, J.C., Finnigan, J.J., 1994. Atmospheric Boundary Layer Flows - Their Structure and Measurement. Oxford University Press. 289 pp.

Krogstad, P.A., Antonia, R.A., 1999. Surface roughness effects in turbulent boundary layers. Exp. Fluids 27, 450-460.

Malhi, Y., 1996. The behaviour of the roughness length for temperature and heterogeneous surfaces. Quart. J. Roy. Meteorol. Soc. 122, 1095-1125.

Panofsky, H.A., Townsend, A.A., 1964. Change of terrain roughness and the wind profile. Quart. J. Roy. Meteorol. Soc. 90, 147-155.

Patel, V.C., 1965. Calibration of the Preston tube and limitations on its use in pressure gradients. J. Fluid Mech. 23, 185-208.

Perry, A.E., Joubert, P.N., 1963. Rough wall boundary layers in adverse pressure gradients. J. Fluid Mech. 17, 193-211.

Perry, A.E., Schofield, W.H., Joubert, P.N., 1969. Rough wall turbulent boundary layers. J. Fluid Mech. 37, 383-413.

Smits, A.J., Wood, D.H., 1985. The response of turbulent boundary layers to sudden perturbations. Ann. Rev. Fluid Mech. 17, 321-358.

Townsend, A.A., 1965. The response of a turbulent boundary layer to abrupt changes in surface conditions. J. Fluid Mech. 22, 799-822. 